\title{
Trivium
}

Revue franco-allemande de sciences humaines et sociales - Deutsch-französische Zeitschrift für Geistesund Sozialwissenschaften

$10 \mid 2012$

Lisibilité

\section{Trace et aura. Remarques à propos du Livre des passages de Walter Benjamin}

\section{Hans Robert Jauss}

Traducteur : Hélène Trespeuch

\section{CpenEdition}

Journals

Édition électronique

URL : http://journals.openedition.org/trivium/4132

DOI : $10.4000 /$ trivium.4132

ISSN : 1963-1820

Éditeur

Les éditions de la Maison des sciences de l'Homme

Référence électronique

Hans Robert Jauss, "Trace et aura. Remarques à propos du Livre des passages de Walter Benjamin », Trivium [En ligne], 10 | 2012, mis en ligne le 30 mars 2012, consulté le 07 septembre 2020. URL : http:// journals.openedition.org/trivium/4132; DOI : https://doi.org/10.4000/trivium.4132

Ce document a été généré automatiquement le 7 septembre 2020

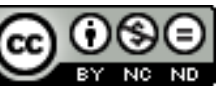

Les contenus des la revue Trivium sont mis à disposition selon les termes de la Licence Creative Commons Attribution - Pas d'Utilisation Commerciale - Pas de Modification 4.0 International. 


\title{
Trace et aura. Remarques à propos du Livre des passages de Walter Benjamin
}

\author{
Hans Robert Jauss \\ Traduction : Hélène Trespeuch
}

\section{NOTE DE L'ÉDITEUR}

Nous remercions Madame Helga Jauss-Meyer de nous avoir accordé l'autorisation de traduire ce texte pour le présent numéro.

« La trace est l'apparition d'une proximité, quelque lointain que puisse être ce qui l'a laissée.

L'aura est l'apparition d'un lointain, quelque proche que puisse être ce qui l'évoque. Avec la

trace, nous nous emparons de la chose ; avec l'aura, c'est elle qui se rend maîtresse de nous ${ }^{1}$."

$(\mathrm{M} \mathrm{16a}, 4)$

\section{I.}

1 S'il est permis au lecteur du Livre des passages d'associer ses réflexions à l'un de ses passages-clé favoris, alors je tiens à faire valoir ce droit à propos de cette note de Benjamin sur « la trace et l'aura ». Ici, comme souvent, la forme de l'aphorisme s'avère particulièrement heureuse, puisqu'elle réunit dans une précise concision et une langue élégante la surprise d'une prise de conscience éclairante et la perspicacité d'une définition, qui pourtant - dans une coincidentia oppositorum non élucidée - allègue la profusion de significations inhérente à l'image dialectique moins comme une réponse que comme une réorientation de la question. Autant que je puisse en juger, Benjamin 
n'a pas particulièrement développé cette idée de la relation dialectique entre la trace et l'aura, si capitale en termes de diagnostic sur l'histoire et le temps présent au XIX siècle. En effet, dans ses notes sur le contenu social de la trace, comme dans ses esquisses d'une théorie de l'aura, c'est précisément l'antagonisme dont il s'agit ici qui, selon moi, fait défaut. On peut même se demander si cela n'explique pas certaines contradictions qui ressortent des notes de Benjamin et de ses tentatives de synthèse qui invitent justement à poursuivre les ébauches interrompues.

2 Au phénomène de la trace doit avant tout être rattaché le flâneur que Benjamin est, il est vrai, le premier à avoir identifié comme une figure spécifique du XIXe siècle. Alors que le promeneur, son précurseur du XVIII siècle (M 13a, 3), découvrait dans la nature sauvage un paysage esthétique, le flâneur découvre un monde qui lui y est opposé, un monde étranger à la nature: "ce dernier voit la ville se scinder en deux pôles dialectiques. Elle s'ouvre à lui comme paysage et elle l'enferme comme chambre ${ }^{2}$ (Stube)» (M 1, 4). La masse des grandes villes est l'élément vital du flâneur. Elle est pour lui tout à la fois labyrinthe et asile, enivrant élixir de vie et incomparable champ d'observation sur lequel son regard physiognomonique fait ses preuves dans l'art de "déchiffrer sur les visages la profession, l'origine et le caractère ${ }^{3}$ " $(\mathrm{M} \mathrm{6,6)}$, enregistrant les traces, perçant ce que la vie publique tient caché. Le flâneur préfigure ainsi le détective (M 13a, 2). C'est l'expérience moderne de l'anonymat au sein de la masse des grandes villes en perpétuelle croissance (attestée depuis $1798^{4}$ ) que cette figure incarne. En littérature, elle se traduit par l'avènement du roman policier dont le contenu social tient, à l'origine, à « l'effacement des traces de l'individu dans la foule de la grande ville ${ }^{5}$ ». Cette perspective littéraro-historique n'est pas propre à Benjamin. À la même époque, on la retrouve en effet chez Roger Caillois. Dans son article pionnier «Paris, mythe moderne » (1937), il livre une interprétation sociale de la manière dont le roman policier moderne a remplacé le roman d'aventures romantique, attirant ainsi l'attention sur les Mohicans de Paris, un modèle du genre qui connut un grand succès en transposant la série de Cooper, Bas-de-Cuir, dans le décor de la «savane de la grande ville». La réception de Poe par Baudelaire, à laquelle Benjamin rend longuement hommage ${ }^{6}$, succède à celle de Cooper par Balzac, par Alexandre Dumas, Eugène Sue et à celle d'autres auteurs du roman noir dans lequel la topographie mythologique de Paris s'empare de la «Babylone moderne » aux alentours de 1840 : «à la Cité innombrable s'oppose le Héros légendaire destiné à la conquérir7. »

3 Le projet de Caillois avait la même ambition que celle qui s'imposa à Benjamin lorsqu'il envisagea son livre des passages: faire une "préhistoire du XIX siècle", comprise comme archéologie mythique de la modernité ${ }^{8}$. À la différence de Benjamin, Caillois voyait néanmoins la genèse du nouveau mythe de la métropole comme le produit actif de l'imagination qui renonce à l'autonomie esthétique - l'aura de l'art idéaliste - dans le but de se réapproprier la réalité aliénée, sur les traces d'une " traduction légendaire de la vie extérieure ${ }^{9}$ " (Baudelaire). Dans le Salon de 1846 de Baudelaire, le chapitre « De l'héroïsme de la vie moderne " apporte la preuve que son auteur établissait dans cette même perspective une rupture radicale entre les nouveaux héros d'une «Iliade de la vie moderne" - l'aristocratie secrète des Vautrin, Rastignac, Birotteau - et le défaitisme de leurs prédécesseurs romantiques, entre l'énergie, l'exigence de pouvoir des uns et le mal du siècle des René, Adolphe ou Obermann. Benjamin souhaitait cependant suivre les pas de Baudelaire dans une autre direction : il cherchait lui aussi à se saisir du nouveau mythe de la modernité, mais en s'intéressant avant tout à la figure 
du flâneur, en qui s'incarne la nouvelle expérience de la masse propre aux grandes villes : « La masse chez Baudelaire. C'est un voile qui se pose devant le flâneur ; elle est la toute dernière drogue du solitaire. - Elle efface, en deuxième lieu, toutes traces de l'individu : elle est le tout dernier asile du réprouvé. - Enfin, elle est, dans le labyrinthe de la ville, le tout dernier labyrinthe, et le plus impénétrable. Grâce à elle, des traits chthoniens jusqu'ici inconnus s'impriment sur l'image de la ville ${ }^{10}$.» $(M 16,3)$ Pourtant, l'ambition qu'a Walter Benjamin, dans le sillon de la critique des idéologies, de prouver qu'une nouvelle aura, illusoire - celle de "l'âme de la marchandise ${ }^{11}$ » - s'est emparée du flâneur, le conduit, lors de l'élaboration de son chapitre sur le flâneur, à se contredire $^{12}$. Étant donné que le flâneur est à peine thématisé dans Les Fleurs du Mal (on pourrait plutôt le déceler dans Le Spleen de Paris, plus proche des physiologies), Benjamin est contraint de s'appuyer principalement sur l'essai de Baudelaire sur Constantin Guys. Or dans le chapitre "L'Homme de la foule ${ }^{13}$ ", Baudelaire refuse d'adopter la perspective propre à la critique des idéologies - qui s'exprime en ces termes chez Engels: "Cette indifférence brutale, cet isolement insensible de chaque individu au sein de ses intérêts particuliers, sont d'autant plus répugnants et blessants que le nombre de ces individus confinés dans cet espace réduit est plus grand ${ }^{14}$.» $\mathrm{Ce}$ jugement d'Engels sur l'agitation des rues londoniennes est en complète contradiction avec l'interprétation que fait Baudelaire de l'«homme de la foule». Si, à propos de Guys, est mise en valeur l'attitude de l'« observateur passionné », célébré en tant que " moi insatiable du non-moi, qui, à chaque instant, le rend et l'exprime en images plus vivantes que la vie elle-même, toujours instable et fugitive ${ }^{15}$ ", ce n'est pas simplement un symptôme, mais déjà la réponse que fait Baudelaire à l'aliénation de la vie dans les grandes villes.

4 L'affirmation de Benjamin selon laquelle le flâneur ne brise ici "qu'en apparence " l'isolement insensible de l'individu dans la foule - à savoir dans la fausse conscience d'une empathie enivrée avec l'âme des marchandises - ne peut donc être justifiée qu'à travers une substitution allégorique du produit de consommation à autrui (Mitmensch) ${ }^{16}$. Qui plus est, cette affirmation se trouve en contradiction avec celle qu'il fait ultérieurement, selon laquelle Baudelaire, à la différence de Hugo, aurait été le gardien du seuil « qui sépare l'individu de la foule ${ }^{17}$ ». Peut-on sérieusement maintenir que Baudelaire, malgré cela, "ne voyait pas au travers de l'illusion sociale qui se cristallise dans la foule ${ }^{18} »$ ? Ou - comme c'est le cas plus tard dans "Sur quelques thèmes baudelairiens "-, que "nous ne pourrons le suivre " lorsqu'il assimile l'«homme de la foule» au type du flâneur ${ }^{19}$ ? Ce qui, aux yeux de Baudelaire, différenciait le flâneur du simple badaud était, entre autres, le fait qu'en tant qu'homme de la foule, il pouvait tout aussi bien rester dans une distance souveraine à l'égard des foules des grandes villes («L'observateur est un prince qui jouit partout de son incognito ») ou y plonger comme dans un «immense réservoir d'électricité » en " amoureux de la vie universelle», tout en mettant en mots la connaissance, sinon la conscience qu'il avait d'elle (" On peut aussi le comparer, lui, à un miroir aussi immense que cette foule; à un kaléidoscope doué de conscience $\left.{ }^{20} »\right)$. À un moment au moins, Benjamin reconnaît que le flâneur dans la foule peut sans doute avoir vu au travers de l'illusion sociale. "L'oisiveté du flâneur est une protestation contre la division du travail $^{21} »(\mathrm{M} 5,8)$ - une supposition qui aurait pu être largement confirmée par une preuve historique : le tableau physiologique intitulé «Le flâneur à Paris » dans Paris ou le livre des cent-et-un (1932). Dans cet ouvrage, il revient au flâneur du XIX ${ }^{e}$ siècle, célébré comme « la plus haute expression de la civilisation moderne », le rôle de satisfaire un 
besoin primordial de son époque - une tâche qui, jadis, incombait au poète ou au philosophe, mais que ces derniers ne sont plus en mesure d'honorer dans la société contemporaine. Alors que chacun, dans l'existence (Dasein) de la foule, ne se préoccupe plus que de son gagne-pain et remarque à peine qu'il a un voisin ou un interlocuteur, seul le flâneur peut - à l'encontre des «effets de la division du travail» - rester "maître de son temps et de lui-même", mener une existence non divisée, tout en restant le véritable point central de la foule : «tout l'intéresse, tout est pour lui un texte d'observation (...) Que de choses il vous apprend! Sous quel aspect inattendu s'offre à vos yeux, avec un pareil démonstrateur, le panorama mobile qui vous environne! Chaque passant a son nom; chaque nom, son anecdote ${ }^{22}$ ».

Il est frappant de constater que Benjamin n'est pas prêt à conférer à la littérature panoramique des physiologies une réelle signification pour la protosociologie du xixe siècle : "ils [les physiologues] sont anodins et d'une parfaite bonhomie ", «ils représentaient, si l'on peut dire, les œillères de l'“animal borné des villes" dont parle Marx quelque part "; « la chose la plus évidente à faire était de donner aux gens une image sympathique des uns et des autres ${ }^{23}$. $\gg$ À ceci on pourrait objecter aujourd'hui que, pour l'Histoire des mœurs de sa Comédie Humaine, Balzac - et ce n'est pas le moindre - alargement exploité ce répertoire incomparable de l'expérience du quotidien que constitue la tradition des Tableaux de Paris (comme l'a montré KarlheinzStierle), condensant la physionomie morale de la grande ville à évolution rapide, pour la transformer en mythes du quotidien ${ }^{24}$. À cela s'ajoute le fait que Le Spleen de Paris de Baudelaire, précisément parce que ce cycle de poèmes en prose dépasse les barrières poétiques des Fleurs du Mal («Les Yeux des pauvres», par exemple, accusant l'insensibilité sociale des amants) s'est lui aussi inspiré des formes de la physiologie moralisatrice et les a perfectionnées ${ }^{25}$. Une pièce comme «Les Foules » (XII) prouve que le poète, en tant que flâneur qui cherche son dernier asile dans la foule humaine, peut voir la grande ville avec «le regard de l'homme aliéné26", mais sait aussi trouver dans la masse son «bain de multitude » : « Multitude, solitude : termes égaux et convertibles par le poète actif et fécond ${ }^{27}$ ». S'il faut trouver un endroit où les « œillères de l'animal borné des villes » sont tombées et où il n'est plus de raisons de discréditer le flâneur en tant que « virtuose de cette identification » à la valeur d'échange, alors c'est bien ici ${ }^{28}$ (M 17a, 2). Si Marx, relativement tôt déjà, lorsqu'il attaqua les Mystères de Paris, "s'assigna pour tâche de faire surgir de cette masse amorphe - qu'un socialisme à nobles intentions s'efforçait d'amadouer - celle d'un prolétariat d'airain ${ }^{29}$ ", ne fallait-il pas alors, au préalable, que soient surmontées l'angoisse et l'horreur provoquées par la foule des grandes villes ${ }^{30}$ et que soit recherchée cette " universelle communion ${ }^{31}$ " que postulait Baudelaire, en mettant au même niveau le flâneur, le poète et l'homme de la foule?

\section{II.}

6 Si l'on prend au mot l'aphorisme de Benjamin sur la trace et l'aura, il invite à envisager le processus accéléré de la révolution industrielle du XIX siècle comme l'« apparition d'une proximité » spécifique à cette époque, qui rendait urgent de poursuivre, dans le négatif de l'ensemble, la trace sur laquelle les arts, sous leurs nouvelles formes d'art social et d'art industriel, tentaient à nouveau de s'emparer de la chose - de l'expérience de plus en plus faible dans un monde vécu (Lebenswelt) dénaturé. En vis-à-vis, en tant 
qu'«apparition d'un lointain", se situerait alors l'aura de l'art autonome, dont Benjamin fut le premier à identifier et décrire efficacement le destin à l'apogée du capitalisme - son déclin augmentant à mesure que progressaient l'art marchandise et le musée imaginaire. Ainsi définie, cette dialectique de l'expérience esthétique du $\mathrm{XIX}^{\mathrm{e}}$ siècle n'est en réalité parachevée ni dans le Livre des passages, ni dans les autres essais rédigés par Benjamin : la théorie de l'art auratique reste emplie de contradictions. Son destin dans la modernité post-romantique est enregistré côté perte en tant qu'histoire d'un déclin, tandis que, côté bénéfice, la page reste quasi vide. Les évolutions opposées d'une esthétique ayant perdu son autonomie, ne pouvaient pas encore être appréciées dans leur intention fondamentale d'humaniser le matérialisme du développement industriel par le beau, parce que Benjamin voyait de prime abord leur fonction sociale marquée par la tare d'une "servitude» (par exemple, A 1a, 8 ; A 2, 2) - ce qui paradoxalement présupposait la permanence du paradigme idéaliste de l'autonomie esthétique.

7 "Définir l'aura comme "l'unique apparition d'un lointain, si proche soit-il", c'est exprimer la valeur cultuelle de l'œuvre d'art en termes de perception spatiotemporelle ${ }^{32}$ ». Est-ce à dire que la détermination centrale de l'art auratique serait, en fin de compte, empruntée à l'expérience du sacré (au caractère inapprochable du tableau de culte ${ }^{33}$ ) ? Dans ce cas, l'approche benjaminienne risquerait de tomber dans le piège d'une théorie de la sécularisation qui, selon la critique formulée à son égard par Hans Blumenberg ${ }^{34}$, est loin d'être incontestable. En prenant comme point de départ le tableau de culte, face auquel l'expérience sacrée et l'expérience esthétique apparaissent encore conjointes, Benjamin se met en difficulté par rapport à la genèse historique de la poésie.

De fait, si dans l'art ancien pré-autonome l'œuvre d'art plastique était liée de manière à ce point évidente à sa place dans la vie que "l'unicité de son existence au lieu où elle se trouv[ait ${ }^{35}$ » était enregistrée dans son aura comme apparition de son lointain, la situation est bien différente dans le cas d'une œuvre de poésie. Car celle-ci reste peu attachée à l'époque, au lieu et à la situation qui la virent naître. Son autonomie sémantique, sa capacité à parler au-delà de son existence unique dans son monde originel, a toujours constitué son caractère esthétique ${ }^{36}$. C'est pourquoi Benjamin était obligé de faire un pas supplémentaire et de remplacer le substrat cultuel de la poésie, qui faisait défaut, par un substrat subjectif de l'esthétique de la production : «l'unicité du créateur ", à savoir l'authenticité de sa production. « Avec la sécularisation de l'art, l'authenticité devient le substitut de la valeur cultuelle ${ }^{37}$ ». Benjamin a présupposé implicitement le processus historique que cette thèse (dissimulée dans une note) devrait impliquer, mais ne l'a pas exposé. S'il avait essayé de le faire, il aurait été confronté au fait que la norme esthétique de l'authenticité (qui reviendra dans la Théorie de l'agir communicationnel de Jürgen Habermas, en tant qu'énoncé expressif réclamant la "transparence d'autoprésentation ${ }^{38}$ »), devrait se trouver, d'un point de vue historique, liée à la phase de l'individualisme militant de l'ère bourgeoise. Avant, elle ne peut être démontrée ; après, elle incombe à la critique du sujet autonome et ne peut alors que caractériser un épisode de l'histoire de l'expérience esthétique ${ }^{39}$. Benjamin remarque lui-même la chose suivante: "En réalité, à l'époque où elle fut faite, une Vierge du Moyen Âge n'était pas encore "authentique", elle l'est devenue au cours des siècles suivants, et surtout peut-être au xix ${ }^{40} \%$. Dès lors la thèse selon laquelle « le hic et nunc de l'original constitue ce qu'on appelle son authenticitée ${ }^{41}$ » ne 
peut être généralisée à l'histoire des arts. Le hic et nunc de la Vierge définissait son aura en tant que valeur cultuelle, qui exclut per se la question de l'authenticité. D'un autre côté, l'aura de l'authenticité qu'a acquis le Faust en tant que création unique de Goethe ne dépendait sûrement plus de l'hic et nunc de la première représentation donnée à Weimar. Comment et par quelle entremise l'authenticité a-t-elle pu se substituer à la valeur cultuelle ? À qui sert donc l'hypothèse selon laquelle l'aura de l'art autonome aurait été empruntée à l'aura du sacré ?

Le concept d'aura chez Benjamin semble par conséquent acquérir sa force herméneutique à partir du moment où il s'éloigne de la thèse non avérée de la sécularisation et se comprend comme une donnée émanant de l'esthétique de la réception, nécessairement rétrospective - l'œuvre d'art comme "apparition d'un lointain, quelque proche que puisse être ce qui l'évoque». Cela est directement explicité dans le célèbre passage de Hegel que cite Benjamin : «Le bel art (...) est né dans l'Église même (...), encore que l'art soit déjà sorti du principe de l'art ${ }^{42}$.» Chez Hegel en effet, le beau dans le tableau de culte n'est justement pas associé à sa valeur cultuelle : très tôt déjà, la piété a eu besoin d'images servant d'objets de dévotion, "mais elle n'avait en aucun cas besoin d'images belles, qui la gênaient même ${ }^{43}$ ». Par conséquent, le beau, même s'il fut d'abord perçu dans le tableau de culte, découle toujours de la négation de sa valeur cultuelle, c'est-à-dire du fait que, dans le temps de la réception, celui qui regarde ne se soumet plus au rituel de la prière. Finalement, lorsqu'un spectateur moderne ressent encore la beauté de l'œuvre d'art classique en tant que valeur cultuelle, l'attitude de profonde contemplation qu'implique cette situation est elle-même déjà une sacralisation secondaire de cette aura de l'authenticité, dont le génie original de l'idéalisme allemand, comme individu autonome, se targuait d'être le créateur. Le culte de l'individu prit la place du culte religieux dans un acte de provocation; s'il doit être question ici de "sécularisation provocatrice ", alors cela ne peut seulement signifier que l'aura de l'œuvre autonome n'est pas née d'un emprunt direct à quelque chose d'existant, mais plutôt qu'elle a été engendrée et légitimée en tant que monde propre au poète qui, à la manière de Prométhée, se pensa comme un autre dieu. Par la suite, seule une réception régressive a voulu voir dans cette émancipation des beaux-arts de leur servitude séculaire émancipation dont l'esthétique des Lumières fut le point culminant -, un succédané d'une religion de l'art, sans prendre en considération le fait que, comme Hegel le rappelait, «nous n'en sommes plus à pouvoir vénérer religieusement les œuvres d'art et à leur vouer un culte ${ }^{44} »$.

Manifestement, sur la route du développement historique des arts, on ne peut définir de manière unanime l'aura de l'œuvre d'art par son autonomie, parce qu'il est impossible de fonder le fait esthétique (das Ästhetische) per se sur la valeur cultuelle : il la transcende, dès le début, et cela se perpétue à travers une opposition à toute forme de sacralisation de l'art. C'est la raison pour laquelle la définition fondamentale de l'aura chez Benjamin - qu'il diagnostiqua d'abord, de manière révélatrice, dans sa phase de disparition - comporte une tonalité nostalgique évidente. En outre, par "l'apparition unique d'un lointain, quelque proche que puisse être ce qui l'évoque ", elle peut signifier quelque chose de toujours différent : le succédané du sacré, le hic et nunc de l'original, le "témoignage historique de la chose ${ }^{45}$ ", l'authenticité de la création subjective, le semblant d'autonomie de l'œuvre d'art ${ }^{46}$, et finalement - en lien cette fois avec la relation de l'homme à la nature - les yeux levés : «Sentir l'aura d'une chose, c'est lui conférer le pouvoir de lever les yeux ${ }^{47} »$. Cette belle métaphore, 
construite en dialogue avec les vers de Baudelaire sur le temple de la nature ("L'homme y passe à travers des forêts de symboles / Qui l'observent avec des regards familiers ») est pourtant trop belle pour être ici encore historiquement vraie, c'est-àdire pour être encore valable en tant qu'index esthétique de la modernité naissante. Car dans le poème Correspondances, Baudelaire avait déjà marqué la limite entre l'expérience auratique de la nature propre au romantisme et le lointain de la nature caractéristique d'une nouvelle ère de la poésie dans laquelle «le déclin de l'aura s'est inscrit $^{48}$ ». Dans le poème lui-même, le regard levé de la nature renaissante est une nouvelle fois mentionné, comme un adieu permettant d'ouvrir la poésie à une nouvelle expérience. Celle de la synesthésie - une perception esthétique qui découvre les correspondances entre les odeurs, les couleurs et les tons « qui chantent les transports des esprits et des sens ", et qui ainsi peut laisser derrière elle les anciens symboles d'une compréhension anthropocentrique de la nature. Pour sauver son concept d'aura pour la modernité, Benjamin a donc été jusqu'à substituer aux «correspondances naturelles » « les trouvailles de la mémoire involontaire », et à la poésie de Baudelaire, la poésie du souvenir de Proust ${ }^{49}$. De la sorte, il voulait défendre un concept d'aura qui devait impliquer dans le souvenir "l'unique apparition d'une réalité lointaine ", et ainsi «le caractère cultuel de l'aura» (à savoir: «le lointain par essence est l'inapprochable $\left.{ }^{50} »\right)$. Il me semble qu'il serait plus cohérent d'attribuer la « donnée de la remémoration ", non à l'aura en voie de disparition, mais plutôt à la trace émergente. Car le lointain essentiel, chez Proust, n'est plus une donnée inapprochable qui se dérobe dans le caractère intemporel du tableau de culte, mais l'inapprochable d'un temps vécu qui se dérobe à la mémoire volontaire. C'est ce qui tombe dans l'oubli, ce qui est enfermé et ainsi conservé. C'est ce dont seul le souvenir involontaire peut s'emparer en ouvrant une porte sur le passé dans l'inattendu " maintenant de la connaissabilité ", et en permettant de reconnaître la trace de la réalité perdue sur laquelle peut dès lors se lancer le narrateur, dans l'aura de l'authentique.

L'aura de la poésie du souvenir que découvre Proust, arrachée à l'inconscient, est directement opposée à l'aura de l'anamnèse platonicienne, tributaire de la réflexion. Elle est née d'une dialectique de la trace et de l'aura que Benjamin ne fait apparaître que là où l'art a complètement « quitté le domaine de la "belle apparence" ${ }^{51}$ » - dans le cinéma, en tant qu'art spécifique à l'ère de la reproductibilité technique. C'est ici enfin qu'un bénéfice peut être tiré de la destruction de l'aura, de la rupture du charme cultuel et de la suppression de l'autonomie esthétique : la déritualisation accomplie de l'art rend le spectateur, jusqu'alors solitaire, disponible pour une réception collective. Benjamin attendait de ce public tout à la fois une attitude critique et de plaisir ${ }^{52}$, et n'en escomptait rien de moins que "l'illumination profane ${ }^{53}$ " de s'approprier soi-même la chose : le devenir pratique de l'expérience esthétique. Néanmoins, l'alternative fatale entre l'esthétisation de la politique et la politisation de l'art pourrait, dans la conclusion appellative de son article sur l'œuvre d'art, ne pas avoir été le dernier mot de Benjamin dans sa dialectique de la trace et de l'aura. L'article intitulé «Sur quelques thèmes baudelairiens » - également achevé en 1939 - entend sauver l'aura du beau et partir à la recherche d'une " expérience qui tente de s'établir sans crises ${ }^{54}$ », expérience à travers laquelle la valeur cultuelle de l'art, recherchée avec une telle obstination, acquiert un sens tout autre, authentiquement profane : "Dans son existence historique, le beau est un appel au rassemblement autour de ceux qui l'ont autrefois admiré. Être saisi par la beauté, c'est, comme le disaient les Romains de la mort, ad plures ire. (...) L'admiration récolte ce que les générations antérieures ont admiré dans l'œuvre ${ }^{55}$. » Je 
reviendrai plus loin sur les implications herméneutiques de cette philosophie de l'histoire esthético-théologique.

III.

"Que l'industrie est rivale des arts »: cette affirmation du vers final en exergue de l'introduction du Livre des passages sonne comme une annonce, pourtant elle n'est développée explicitement ni dans les notes, ni dans les essais. Naturellement, l'inestimable documentation compilée traite des grandes performances de la production industrielle : la construction en fer en tant qu'ingénierie, l'utilisation du verre, l'éclairage, la photographie, le graphisme publicitaire, le feuilleton littéraire, les aménagements propres aux expositions, l'haussmannisation. Pourtant les perspectives ainsi ouvertes ne sont pas réunies dans une histoire consacrée à la rivalité entre art industriel, art social et art traditionnel. Dans cette synthèse fragmentaire, Paris, capitale $d u$ XIX ${ }^{e}$ siècle, les passages et les intérieurs, les halls d'exposition et les panoramas sont surtout abordés en tant que "résidus d'un monde de rêve " ("chaque époque rêve la suivante $\left.{ }^{56} »\right)$. Selon Benjamin, ce n'est qu'avec le surréalisme que le regard sur ces éléments fut libéré, car il identifia les « ruines de la bourgeoisie " pronostiquées par Balzac et en détruisit les symboles de ses aspirations ${ }^{57}$. Donnant l'impression que le regard mélancolique a été complètement hypnotisé par la grande idée de la philosophie de l'histoire, celle d'une préhistoire du $\mathrm{xIX}^{\mathrm{e}}$ siècle, et par son corrélat poétique, le moderne comme ère de l'enfer (contre-image dialectique du monde des marchandises saint-simonien), les synthèses ébauchées appréhendent, dans des descriptions souvent brillantes, tout ce qui se laisse inscrire dans la mythographie de la Babylone moderne ou dans la pathographie de la conscience collective en adhésion avec le «caractère fétiche de la marchandise». Dans cette crise de la perception et cette aliénation croissante de l'expérience du monde vécu, ce qui a été fait pour saisir le développement rapide de la civilisation technique dans de nouvelles formes d'art, pour le maîtriser à travers de nouvelles définitions de sa fonction sociale et pour le diffuser à partir des modèles et des expériences de l'ancien art "auratique ", n'est quasiment pas pris en considération. Le développement des forces productives de la modernité au XIX siècle n'a pas seulement "émancipé les formes plastiques de la tutelle de l'art ${ }^{58}$ ». Il a également permis à l'art de sortir du règne autonome de la belle apparence et l'a replacé dans son rôle social - qui ne mérite pas d'être simplement rabaissé à une "servitude». Je souhaite expliciter la trace ainsi sous-estimée d'une expérience esthétique neuve, en phase de construction, et qui, face au processus d'aliénation de la production industrielle des marchandises, serait à même de contrebalancer la perte de l'aura sans participer pour autant de l'idéologie dominante du progrès, à partir de trois exemples.

13 1) Dans sa critique de la conception non dialectique, chez Benjamin, du «caractère de fétiche de la marchandise ", Adorno relève le "passage sur la "libération accordée aux choses de la corvée d'être utiles" » en le présentant comme « le génial tournant à même de sauver dialectiquement les marchandises ${ }^{59}$ ", qu'il aurait souhaité voir davantage développé. Ce passage se situe dans un chapitre sur les intérieurs, que Benjamin a su appréhender dans la signification symptomatique qu'ils représentaient par rapport aux bouleversements du monde vécu (Lebenswelt), et ce jusqu'au Jugendstil. Et il le fait de telle manière que ce passage apparaît comme l'un des moments phares de son projet: 
«Pour le particulier les lieux d'habitation se trouvent pour la première fois en opposition avec les lieux de travail. Ceux-là viennent constituer l'intérieur (...). Le particulier qui ne tient compte que des réalités dans son bureau demande à être entretenu dans ses illusions par son intérieur. (...) Celui-ci représente pour le particulier l'univers. Il y assemble le lointain et le passé. Son salon est une loge au théâtre du monde ${ }^{60}$. $"$ Alors que l'importance grandissante du musée imaginaireest ici diagnostiquée in nuce, mais encore de manière péjorative, un début de trace à même de compenser la perte de l'expérience auratique commence du côté du collectionneur. Pour ce dernier, l'intérieur est l'asile où se réfugie l'art : «Le collectionneur se plaît à susciter un monde non seulement lointain et défunt mais en même temps meilleur ; un monde (...) où les choses sont libérées de la corvée d'être utiles ${ }^{61}$.» Malheureusement, dans l'interprétation de son propre aphorisme, Benjamin dément presque cette ébauche prometteuse qui aurait pu le conduire à une appréciation plus juste de l'« esthétisme » tant vilipendé. Il croit devoir critiquer le collectionneur parce qu'il ne confère aux choses qu'il idéalise « que la valeur qu'elles ont pour l'amateur au lieu de la valeur d'usage ${ }^{62}$.» Pourtant, ce n'est pas avec l'appréciation des choses selon leur valeur d'usage (qui d'après Adorno " ne renvoie qu'au stade de la division du travail ${ }^{63}$ ») que commence leur libération du statut de marchandises, mais avec la valeur que leur confère l'amateur, déchargée de toute utilité (voir H 3a, 1). C'est ce que montre précisément l'œuvre d'art elle-même qui en tant que marchandise sui generis échappe toujours aux « lubies théologiques » de la valeur d'usage et de la valeur d'échange.

Ailleurs au contraire, Benjamin considère le fait de collectionner comme "diamétralement opposé à l'utilité » et le place dans la «catégorie remarquable de la complétude». Il s'agirait d'« une tentative grandiose pour dépasser le caractère parfaitement irrationnel de la simple présence de l'objet dans le monde, en l'intégrant dans un système historique nouveau, créé spécialement à cette fin, la collection ${ }^{64}$. 》 (H 1a, 2) La complétude comme idéal de l'art de collectionner intègre l'arbitraire de la tradition dans un nouveau rapport: un système historique qui garde en même temps dans l'ordre du souvenir l'arbitraire de la réalité passée (H 2, 7). Collectionner comme "forme de ressouvenir pratique », ce n'est donc pas restituer précisément l'auratique comme il a effectivement été, mais suivre la trace de l'appropriation pour conduire le lointain dans la manifestation « la plus convaincante ${ }^{65}$ » de la proximité (H 1a, 2). Ceci explique une réflexion de Benjamin très significative, et dans un premier temps surprenante: «La vraie méthode pour se rendre les choses présentes consiste à se les représenter dans notre espace (et non à nous représenter dans le leur). (...) Il en va de même, en vérité, avec la contemplation de grandes choses du passé, comme la cathédrale de Chartres, ou le temple de Paestum, quand elle est couronnée de succès : elle consiste à les accueillir dans notre espace. Ce n'est pas nous qui entrons en elles, ce sont elles qui entrent dans notre vie ${ }^{66}$.» (H 2, 3; Premières notes, $\left.\mathrm{I}^{\circ} 2\right)$ La «vraie méthode " par conséquent, l'appropriation, est donc opposée à l'empathie de l'historicisme: c'est à lui qu'est fait le reproche de la fausse conscience, et non au musée imaginaire que le collectionneur s'est construit avec le «regard du grand physionomiste ${ }^{67} »(\mathrm{H} 2,7)$.

15 2) En exploitant les documents sur la construction en fer et les aménagements propres aux expositions, Benjamin voulait approfondir une thèse de Marx selon laquelle « à l'origine, la vieille forme du moyen de production influe sur la forme nouvelle » (F 2a, 5) dans le sens où ce processus aurait suscité, dans la conscience collective, des images de souhait (Wunschbilder) «où le nouveau et l'ancien se compénètrent ${ }^{68}$ ». Si, à ses 
débuts, la production industrielle était " prisonnière du rêve ${ }^{69}$ ", en particulier lors du développement triomphal des formes sœurs de la fonte et du verre (F 1a, 2), les rêves des ingénieurs et des architectes n'étaient pour autant nullement régressifs. Le style empire considérait moins la nouvelle technique de la construction en fer comme « une contribution au renouveau de l'architecture dans l'esprit de la Grèce antique ${ }^{70} »$ que comme une surenchère par un « autre art » déterminé à s'« élever aussi loin du système médiéval que le système de l'art éleva le Moyen-Âge au-dessus du système du linteau monolithe de l'Antiquité ${ }^{71} »$ (citation de Boetticher, F 1, 1). Dans la relation entre art et industrie, le fait de prélever le nouveau sur de l'ancien peut, sous l'apparence d'une " compénétration ${ }^{72}$ » continue, relever de motivations totalement différentes. Tant que le caractère fonctionnel du fer n'est pas reconnu, la forme ancienne peut encore, dans un premier temps, se greffer sur la nouvelle fonction (par exemple les wagons de chemin de fer en forme de diligences, F 7, 3). L'ancienne et la nouvelle fonction peuvent se retrouver dans un objet hybride. C'est le cas du passage qui, en tant que construction en fer «se trouve déjà, du point de vue fonctionnel, dans le domaine de l'espace en largeur (Breitraum), mais du point de vue architectural, (...) est encore du côté de l'ancienne "halle" ", et conserve ainsi quelque chose de sacré (" un souvenir de la nef » encore attaché « à cette enfilade de marchandises qu'est le passage ${ }^{73}$ », F 4, 5). Le lieu de production de la nouveauté peut également citer d'anciennes formes, pour exorciser l'étrangeté du monde industriel et lui donner un aspect davantage familier (des usines avec des galeries et, à l'intérieur, des escaliers tournants en fer, F 5a, 6). Une fois devenue art industriel pleinement épanoui, la nouveauté peut enfin intégrer la beauté de la nature, dépassée, pour la conserver comme le ferait un musée (comme les célèbres ormes sous la voûte centrale du palais de cristal, qui renvoie à la forme nouvelle de la serre, F 4, 1 ; F 4, 2). Mais l'art industriel autonome peut aussi mettre la nouveauté à la place de l'ancien art sacré, de manière provocatrice et triomphante, comme dans le cas de l'exposition universelle de Paris en 1855 : «Quatre locomotives gardaient l'entrée de l'annexe des machines, semblables à ces grands taureaux de Ninive, à ces grands sphinx égyptiens qu'on voyait à l'entrée des temples ${ }^{74}{ }_{\text {» }}(\mathrm{G} 8 \mathrm{a}, 2)$.

16 3) Ce que Benjamin dit des expositions universelles peine à satisfaire le lecteur contemporain : selon lui, elles auraient été, tout au plus, « les centres de pèlerinage de la marchandise-fétiche ", "la haute école où les masses exclues de la consommation apprirent l'identification à la valeur d'échange ${ }^{75}$ ». " Tout regarder, ne toucher à rien " $(G 16,6)$ est un verdict - conservateur, en somme - que Georg Maag a dernièrement revu profondément au travers d'une analyse de documents sur la réception de ces expositions. Aux témoignages d'euphorie, suscitée par la surenchère des évolutions industrielles et techniques dépassant toute attente s'opposent ici les symptômes d'une peur insurmontable à l'égard de la supériorité des capacités humaines, captée dans des expériences diverses et des rêves collectifs. En même temps, cette peur donna naissance à une nouvelle esthétique de l'ère industrielle. Le chapitre de Maag sur le "Crystal Palace » met au jour la dialectique - qui fait défaut chez Benjamin - de l'industrie et du rêve : la soudaine transformation de la réalité technique maîtrisée en fantasmagorie, en vieilles promesses de contes et de mythes (construction de la tour de Babel) appelées en renfort. Ces promesses se cristallisent, même chez des écrivains progressistes comme Théophile Gautier, de manière contre-paradigmatique, tout particulièrement autour du nouveau mythe de l'Orient (indien): «L'expérience, qui reste obstruée par l'abstraction des véritables conditions de vie, est récupérée par l'imaginaire ${ }^{76}$. $"$ Je souhaite ici convoquer Baudelaire, un autre témoin important. 
Benjamin a certes cité son compte rendu de l'Exposition universelle de 1855, mais ne l'a pas suffisamment exploité (G 15a, 2). Baudelaire y suggère qu'il est conscient d'être au tournant d'une époque à travers la question rhétorique suivante: qu'est-ce qu'un Winckelmann moderne aurait à dire devant les pièces chinoises exposées, d'une beauté bizarre, déconcertante et aussi fascinante qu'inexpliquée ? Le Winckelmann classique - et avec lui, le canon de l'esthétique autonome, de la philosophie de l'art, ainsi que les normes de l'académisme qui régnait jusqu'alors - échouerait ici inévitablement («tout peuple est académique en jugeant les autres, tout peuple est barbare quand il est jugé ${ }^{77}$ »). Comparer et juger le pluralisme fondamental des produits exposés provenant de toutes les nations nécessite selon lui un revirement radical. Le spectateur critique devrait apprendre à s'approprier ce qu'exclut le canon soi-disant intemporel du beau, et reconnaître dans l'étranger précisément ce qui est bizarre, singulier, la quintessence d'une nouvelle "beauté universelle». Saisir la nouvelle esthétique des expositions universelles, selon lui, ne peut se faire grâce à une éducation historique, elle nécessite au contraire une nouvelle naïveté qui, dans la diversité des produits artistiques, recherche et peut reconnaître le nouveau qu'il n'est possible de déduire d'aucune règle ${ }^{78}$.

La condamnation de l'historicisme et de l'académisme, qui permet d'ouvrir le regard à la beauté universelle et synchrone des arts réunis dans le musée imaginaire de l'exposition universelle, implique pour Baudelaire une véhémente condamnation de l'idée de progrès : «Qui veut y voir clair dans l'histoire doit avant tout éteindre ce fanal perfide ${ }^{79}$ ». À l'adresse du "Français moyen » - lecteur de journaux déjà tellement "américanisé » (s'agit-il d'un néologisme de Baudelaire?) par ses "philosophes zoocratiques et industriels ", qu'il ne serait même plus en mesure de faire la différence entre le monde matériel et le monde spirituel ${ }^{80}$ - ce témoignage aurait très bien pu s'intégrer à l'optique de Benjamin, au même titre que la prise de conscience chez Baudelaire d'un nouveau cosmopolitisme : la reconnaissance d'une «égale utilité » de toutes les nations à l'égard de l' «immense analogie universelle ${ }^{81} »$ de leurs produits. L'autre facette de cet industrialisme n'est donc pas seulement, dans la deuxième moitié du XIX ${ }^{e}$ siècle, la nouvelle esthétique d'une alliance entre art et industrie, mais aussi une nouvelle solidarité des masses qui se manifeste dans l'euphorie (G 10a, 2; G 13a, 1). Car si d'un côté la "fantasmagorie de la culture capitaliste trouva son plus grand épanouissement lors de l'Exposition universelle de $1867^{82}$ ", de l'autre côté les délégations d'ouvriers ont également ouvert la voie dès 1862 - comme Benjamin luimême le remarque - à la fondation de l'Internationale $(\mathrm{G} 7 \mathrm{a}, 3)$.

Selon Benjamin, le développement de la technique (industrielle) jusqu'au début du XIX siècle se serait opéré beaucoup plus lentement que celui de l'art, mais par la suite, la situation se serait renversée et le processus technique aurait désormais dicté son tempo à l'art $(G 1,1)$. Cette remarque donne l'occasion de se rappeler du développement non synchrone des différents arts. Tandis que, dans les périodes anciennes, la poésie fut la première à s'affranchir des fonctions du culte, il semble qu'au XIX ${ }^{e}$ siècle elle repassa derrière les formes artistiques de la production industrielle, celles qui n'avaient plus de valeur auratique et qui triomphaient dans les halls des expositions universelles. Dans cette nouvelle alliance entre art et industrie, la poésie joue à peine un rôle. Hippolyte Babou en témoigne, lorsqu'il s'en plaint en ces termes : "L'art et l'industrie ! Oui, c'est en effet pour eux, pour eux seuls, qu'on a réservé en 1855 cet inextricable réseau de galeries, où ces pauvres littérateurs n'ont pas même obtenu six pieds carrés, la place 
d'une pierre tumulaire ${ }^{83}$ !» (G 16a, 2). Le slogan de l'art pour l'art, compris comme la tentative de "protéger l'art en le rendant imperméable au développement de la technique ${ }^{84}$ » serait resté à cet égard une vaine tentative d'auto-affirmation. Cela peut finalement expliquer pourquoi Baudelaire le condamne en tant que " puérile utopie ${ }^{85}$ ». À la même époque commençait le développement, encore très hésitant, d'une nouvelle poésie de l'industrie. Maag a montré que cette première approche poétique du monde industrialisé restait encore dans une pré-compréhension contrastive de la nature abandonnée par la production industrielle. Dans les Fleurs du mal, l'environnement industriel n'apparaît pas encore. Toutefois, c'est justement Baudelaire qui a préparé la voie à une esthétique de la modernité industrielle, en ne fondant plus sa poésie sur le concept nostalgique de nature issu du romantisme, mais sur l'expérience contradictoire d'un monde devenu abstrait - une expérience dans les abîmes de laquelle plonge le sujet des Fleurs du mal, et ce de manière exemplaire, afin de le célébrer dans ses extases.

IV.

Le fait que le Livre des passages soit riche en examens originaux et en perspectives à développer, - parmi elles figure une histoire de la réception qui a maintenant bien progressé et à laquelle la recherche sur Baudelaire est grandement redevable - ne devrait pas laisser ignorer les raisons pour lesquelles l'une des principales thèses de cet ouvrage met son auteur en difficulté, et ce à propos du principal témoin de sa démonstration : Baudelaire. L'affirmation en question se trouve déjà formulée dans les Premières notes (1927-1929), et est plus tard reprise mot pour mot : "Le "moderne" comme temps de l'enfer. Les châtiments de l'enfer sont toujours à la pointe de la nouveauté dans ce domaine. Il ne s'agit pas de dire que "les mêmes choses sans cesse" arrivent, encore moins de parler ici d'éternel retour. Il s'agit plutôt de ceci : le visage du monde ne se modifie jamais dans ce qu'il y a de plus nouveau, cette extrême nouveauté demeure en tous points identique à elle-même. C'est cela qui fait l'éternité de l'enfer. Déterminer la totalité des traits sous lesquels le "moderne" se manifeste, ce serait donner une présentation de l'enfer ${ }^{86}$. ( (S 1, 5 ; Premières notes, $\left.G^{\circ} 17\right)$ L'étude de la réception contemporaine de l'œuvre de Baudelaire montre que ses amis s'étaient opposés à sa mise en examen pour immoralité, et avaient cherché à disculper l'auteur des Fleurs du mal en le présentant comme le Dante d'une époque décadente ("c'est du Dante athée et moderne, du Dante venu après Voltaire ", J 3a, $1^{87}$ ). Et il est vrai que dans ses « paysages de l'ennui » que Benjamin observe à son tour, une « idylle mortelle ${ }^{88}$ » de la ville à plusieurs facettes se fait jour. Toutefois, cet aspect infernal ne représente en aucun cas l'ensemble des traits caractéristiques de la modernité dans le chef-d'œuvre de Baudelaire. Le lecteur est également guidé à travers un purgatoire moderne ${ }^{89}$ - pour rester dans le paradigme dantesque - et découvre soudain, dans l'hétérogène " paysage de l'extase ${ }^{90} "$, des lieux de repos, ceux du souvenir et des paradis artificiels. En l'occurrence, le revirement de l'expérience de la nouveauté et de l'extrême nouveauté en quelque chose d'identique, de toujours semblable ou d'extrêmement ancien n'est nullement nécessaire (la " préhistoire » recherchée). L'esthétique baudelairienne de la nouveauté affirme ses prétentions morales en prônant une curiositas exploratoire, qui peut certes décevoir, mais est aussi intarissable. Le vers final du dernier poème - «Au fond de l'inconnu pour trouver du nouveau » - devait, selon Benjamin, faire apparaître la nouveauté comme " origine de cette apparence illusoire » et " quintessence de cette fausse conscience ${ }^{91} »$. À l'encontre d'une telle interprétation, on peut dire que 
Baudelaire a ici renouvelé un célèbre paradigme dantesque: le dernier voyage qui conduit Ulysse au-delà des limites imposées à l'homme, aux antipodes de l'ancien monde, insouciant à l'égard du ciel et de l'enfer - que Baudelaire évoque avec provocation: «Nous voulons, tant ce feu nous brûle le cerveau, / Plonger au fond du gouffre, Enfer ou Ciel, qu'importe? » Pour lui, c'est précisément le nouveau qui permet de reconvoquer l'ancien et de ressusciter un passé mort, comme il le montre à travers le paradigme de la mode ${ }^{92}$. C'est l'impulsion première qu'a sous-estimée Benjamin, ainsi que la fonction herméneutique de l'esthétique de la nouveauté avec laquelle Baudelaire accomplit la transition de l'historicisme à l'esthétisme. Celle-ci contredit le concept d'une modernité régressive que développe Benjamin en établissant sa "préhistoire ", dans la mesure où elle correspond à sa théorie du «Maintenant de la connaissabilité ». Benjamin n'y fait pas appel - à ses dépens.

En se lançant dans une " préhistoire du Xix ${ }^{e}$ siècle », Benjamin a suscité chez Adorno le lourd reproche suivant : "La formule d'après laquelle "nouveau et l'ancien se compénètrent" me semble au plus haut point douteuse et ce pour la même raison qui me faisait critiquer l'image dialectique comme régression. Là, on ne se rapporte plus à l'ancien, c'est ce qu'il y a de plus nouveau qui, en tant qu'apparence et fantasmagorie, est lui-même l'ancien ${ }^{93}$ ». Pourtant, de cette critique un noyau de vérité peut être sauvé, si l'on s'engage à reformuler la thèse principale de Benjamin, dans laquelle il récuse l'idée d'une archéologie du xix siècle (la recherche de formes préhistoriques, autrement dit des archétypes de l'inconscient collectif), au profit de l'idée suivante : "Le concept d'une préhistoire du XIX ${ }^{\mathrm{e}}$ siècle n'a de sens que là où le XIX ${ }^{\mathrm{e}}$ siècle serait présenté comme forme originaire de la préhistoire, sous une forme, par conséquent, où l'ensemble de la préhistoire se rassemble à nouveau dans des images qui appartiennent au siècle écoulép. . (N 3a, 2) Au siècle précédent, notre modernité a - telle est ma lecture de ce passage - sa préhistoire spécifique, autrement dit : le XIX siècle, avec la première apparition non maîtrisée de la technique, est l'« antiquité » de notre modernité, en tant que préhistoire de ce qui arrive ultérieurement! La foi naïve dans le progrès, la sensation simplement négative du tout-nouveau, ne serait dans la conscience collective qu'une forme onirique prise par les événements: "Le collectif qui rêve ignore l'histoire. Pour lui, les événements se déroulent selon un cours toujours identique et toujours nouveau. La sensation du tout-nouveau, du tout-à-fait-moderne est une forme $\mathrm{du}$ devenir tout aussi onirique que l'éternel retour du même ${ }^{95}$.» (S 2,1 ; Premières notes, $\left.\mathrm{M}^{\circ} 14\right)$. Le réveil de rêves collectifs, ce moment où l'illusion historique se dissipe serait alors, selon Baudelaire, conscience du temps aiguë, marquée par le moment du réveil - moment qui, selon Benjamin, est identique au "Maintenant de la connaissabilité dans lequel les choses prennent leur vrai visage, leur visage surréaliste ${ }^{96}$ "(N 18, 3; N 3a, 3). Cela ne voudrait pas seulement dire que, dans les images dialectiques des Fleurs du mal, «l'Autrefois rencontre le Maintenant dans un éclair pour former une constellation ${ }^{97} »(\mathrm{~N} 2 \mathrm{a}, 3)$, mais aussi que les Fleurs $d u$ mal permettent de percevoir le présent à partir d'une anticipation du futur - le Paris du temps présent comme ruine de la société bourgeoise et de la culture capitaliste.

21 Si l'on adopte ce type de lecture, il devient inutile de s'acharner à tenter de démontrer, encore et toujours, que le moderne et l'antique (classique) se compénètrent dans les Fleurs du Mal. Devient caduc également le reproche selon lequel l'absence d'une analyse de l'antiquité classique serait le principal point faible de la théorie baudelairienne de la

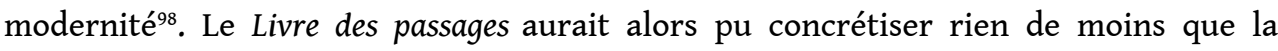


grandiose ambition de Maxime Du Camp « d'écrire sur Paris ce livre que les historiens de l'antiquité n'ont pas écrit sur leurs villes ${ }^{99}$ " (C 4). Dans le chapitre "Le Paris antiquisant, catacombes, démolitions, déclin de Paris », Benjamin a bien mis en valeur le moment où, dans la vie de Du Camp, survient cette inspiration qui donna lieu à son grand livre d'observation, Paris, ses organes, ses fonctions et sa vie dans la seconde moitié du XIXe siècle (6 vol., 1869-1871). Il ne parviendra toutefois pas à l'accomplir, en tant que topographie présupposant le déclin de la métropole (C 4). Alors qu'il attendait la fabrication d'une paire de lunettes et qu'il s'apercevait ainsi pour la première fois de sa situation d'homme âgé avec gravité, Du Camp réfléchissait à « cette loi de l'inévitable destruction qui gouverne toute chose humaine ${ }^{100} »$. Sur le Pont Neuf, il eut l'intuition fulgurante d'écrire ce livre sur Paris, qui serait l'œuvre de sa vieillesse, le couronnement de sa carrière - celle qu'on aurait pu écrire sur Athènes au temps de Périclès, sur Rome au temps de César, ces villes déchues de l'Antiquité. Ici, aux yeux de Benjamin, ce n'est pas seulement «l'inspiration antique » qui est «hautement signifiante", mais surtout le déclin anticipé des métropoles modernes. Benjamin revient sur ce point ultérieurement: "Les rêveries sur le déclin de Paris sont un symptôme du fait que la technique n'était pas acceptée. Elles traduisent la conscience obscure de ce que la croissance des grandes villes s'accompagne de celle des moyens qui permettent de les raser ${ }^{101}$.» (C 7a, 4) Le lecteur d'aujourd'hui pourrait, il me semble, n'attendre rien d'autre de Benjamin qu'il ait justement établi ce précieux examen dans son Livre des passages - avec son sens du diagnostic, incomparable en matière de savoir non encore conscient, refoulé ou oublié dans l'espace mythique des traditions d'une époque -, qu'il l'ait étoffé pour en faire une ligne directrice de sa préhistoire de la modernité à venir. Celui-ci serait devenu la pierre de touche de sa théorie de l'image dialectique, exigeant de la nouvelle méthode dialectique en histoire de "vivre l'Autrefois avec l'intensité d'un rêve pour voir dans le présent le monde éveillé auquel le rêve se rapporte! » (Premières notes, $\left.\mathrm{F}^{\circ}, 6\right)$. Cette " révolution copernicienne ${ }^{102}$ » $(\mathrm{K}$ 1,1 ), avec laquelle « il faut instruire le procès de la contemplation " (Premières notes, $\left.\mathrm{Q}^{\circ}, 6\right)$, ne suppose pas seulement que, dans le "Maintenant de la connaissabilité », le passé soit compris en fonction de ce qui le conditionne dans le présent. Elle implique également que la constellation entre temps présent et histoire devienne perceptible dans le point de rupture que constitue un réveil $(\mathrm{N} 18,4)$ à même de dissiper l'apparence illusoire de la continuité épique (la «tradition des vainqueurs») (Premières notes, $\mathrm{O}^{\circ}$, 81) et de rendre possible sa transformation dialectique en agir politique (Premières notes, $\mathrm{O}^{\circ}, 56$ ). Sur ce point, je souhaite remarquer que le «Maintenant de la connaissabilité » est d'origine esthétique, alors que le "Maintenant du réveil » est d'origine théologique. Et dans ces deux concepts revient l'opposition entre trace et aura sans qu'on puisse y trouver une solution dialectique définitive.

Une image dialectique «est ce en quoi l'Autrefois rencontre le Maintenant dans un éclair pour former une constellation ${ }^{103}$. » $(\mathrm{N} 2 \mathrm{a}, 3)$ Cette définition est ensuite explicitée de la manière suivante: "La marque historique des images n'indique pas seulement qu'elles appartiennent à une époque déterminée, elle indique surtout qu'elles ne parviennent à la lisibilité qu'à une époque déterminée ${ }^{104} »(\mathrm{~N} 3,1)$. Ici, la connaissabilité n'est pas comprise comme lisibilité incidemment, car l'attente suivante : "Chaque moment historique compris comme possibilité libère des possibilités d'histoire. Chaque possibilité ainsi libérée vient à la rescousse de la possibilité du présent ${ }^{105}$ » se concrétise de la manière la plus évidente dans le cas de textes littéraires. Car, l'histoire de leur réception (si elle s'avère conséquente) démontre qu'on ne pouvait interroger ces écrits 
avec n'importe quelle question à n'importe quelle époque. Toute la richesse de leurs significations ne pouvait être reconnue dès le début, elle ne s'est révélée que progressivement - dans la mesure où, au fil de l'expérience et des changements de perspective, et souvent en opposition à la tradition, la signification potentielle de ces textes a toujours été comprise et lue de manière différente, nouvelle. Benjamin a ainsi anticipé l'esthétique de la réception, qui ne s'est imposée que trente ans plus tard. C'est la raison pour laquelle Gerhard Kaiser a remarqué à juste titre, à propos des «Thèses sur la philosophie de l'histoire » et de l'essai sur Fuchs, que « l'idée d'héliotropisme en histoire artistique et culturelle est acquise, dans le champ de la réception des œuvres et des textes ${ }^{106}$ ». En effet, l'espoir qui sommeille dans le passé - « De même que certaines fleurs tournent leur corolle vers le soleil, le passé par un mystérieux héliotropisme, tend à se tourner vers le soleil qui est en train de se lever au ciel de l'histoire ${ }^{107}$ " équivaut à telle signification qui, encore latente dans l'ouvrage, attendrait d'éclore à la lumière d'un ouvrage ultérieur. C'est la raison pour laquelle Benjamin pouvait également écrire : «La réception des grandes œuvres d'art très admirées est un ad plures ire $^{108} »(\mathrm{~N} 7 \mathrm{a}, 4)$, identifiant ainsi l'expérience esthétique à une conception religieuse éloquente (dans la Rome antique, la formule renvoyait aux défunts!). L'origine esthétique de l'exhortation suivante, adressée à l'historien dialecticien, "prendre conscience de la constellation critique que tel fragment du passé forme précisément avec tel présent ", est particulièrement éclairante dans l'essai sur Fuchs. Elle y est introduite à travers l'exemple d'œuvres d'art qui intègrent leur préhistoire et leur fortune (Nachgeschichte), « (...) une fortune en vertu de laquelle leur préhistoire se révèle être elle aussi soumise à d'incessants changements. Elles [ces œuvres] lui apprennent comment leur fonction est capable de survivre à leur créateur et de s'émanciper de ses intentions ; comment l'accueil par les contemporains est un aspect de l'influence que l'œuvre d'art exerce aujourd'hui sur nous, et comment cette influence ne repose pas seulement sur la rencontre avec l'œuvre, mais aussi avec l'histoire qui lui a permis de venir jusqu'à nous ${ }^{109}$. "

23 Cette exhortation, qui anticipe in nuce le programme de la future esthétique de la réception de manière presque parfaite, se laisse également penser à travers la dialectique de l'aura et de la trace comme une "révolution copernicienne», transformant la contemplation en présentification (Vergegenwärtigung). L'attitude contemplative serait alors tournée vers l'apparition d'un lointain, et à travers elle, l'aura se rendrait maîtresse de nous ; l'attitude dialectique, en revanche, s'orienterait vers la présentification productive du lointain, et à travers elle, la "remémoration " chercherait à s'emparer de la chose (constellation critique exigeant une appropriation consciente du passé, sans qu'il suffise que «le présent éclaire le passé. » N 2a, 3). Toutefois, Benjamin n'a pas tiré cette conséquence. Manifestement, il a préféré tenter de conserver dans le "Maintenant de la connaissabilité » un moment auratique. La définition selon laquelle l'image est «ce en quoi l'Autrefois rencontre le Maintenant dans un éclair pour former une constellation» est ici explicitée de manière surprenante, mais surtout difficilement compréhensible : «l'image est la dialectique à l'arrêt » (N 2a, 3). Dans la réception productive des œuvres d'art, la dialectique n'arrive justement pas à un point d'arrêt : le Maintenant de la lisibilité implique encore d'autres lisibilités à venir. Pourquoi l'évidente partialité de toute réception artistique ne seraitelle pas également valable pour l'histoire? Manifestement parce qu'ici, en passant de l'esthétique au politique, un nouveau paradigme théologique est appelé en renfort : le 
«blocage messianique des événements ${ }^{110}$ ». Pourquoi l'histoire doit-elle donc être bloquée, en vertu de quel espoir, à quelle fin?

Les thèses sur la philosophie de l'histoire mettent en évidence le recours à la théologie messianique: "Le passé est marqué par un indice secret, qui le renvoie à la rédemption. (...) Nous avons été attendus sur la terre. À nous, comme à chaque génération précédente, fut accordée une faible force messianique sur laquelle le passé fait valoir une prétention ${ }^{111}$. ( (II) Ici, Benjamin crée un court-circuit entre le paradigme de l'esthétique de la réception et celui de la théologie : la rédemption du passé prend la place de l'œuvre, dont l'interprète rend compréhensible une signification jusqu'alors inconnue pour le présent. La force messianique a cependant plus à apporter que l'interprète. Elle ne doit pas seulement arracher tel ouvrage particulier à l'œuvre d'une vie ou à son époque, détacher une époque déterminée du cours homogène de l'histoire ${ }^{112}$ (XVII) ; elle ne doit pas s'arrêter, «jusqu'à ce que la totalité du passé soit, dans une apocatastase historique, introduite dans le présent ${ }^{113}$.» $(\mathrm{N} 1 \mathrm{a}, 3)$ Cet espoir dépend au préalable de la clause suivante : «Certes, ce n'est qu'à l'humanité rédimée qu'échoit pleinement son passé. C'est-à-dire que pour elle seule son passé est devenu intégralement citable ${ }^{114}$. » (III) Mais, à la fin, cette clause est à nouveau abolie dans une caractérisation accentuée de la coïncidence entre temps présent et histoire : ce n'est pas un état final à venir, mais chaque moment vécu et arrêté qui peut déjà constituer, pour la rédemption de l'humanité, le jour du Jugement dernier (III) ou - comme pour les juifs auxquels il était interdit de sonder le futur - être « la porte étroite par laquelle le Messie pouvait entrer ${ }^{115}$ » (XVIII B).

Si ici - comme le remarque Gerhard Kaiser - la résurrection de l'histoire doit concrétiser un espoir qu'Adorno, Horkheimer, Marcuse et Bloch plaçaient dans une résurrection de la nature déchue ${ }^{116}$, la solution de Benjamin est payée au prix d'une dernière contradiction : l'impossibilité de présenter dialectiquement le «Maintenant de la connaissabilité » et le «moment du réveil ». Le Maintenant de la connaissabilité, dans lequel une époque particulière du passé refoulé atteint la lisibilité, suppose un accès conscient, et donc l'appréhension de la trace pour se rendre maître de la chose (de l'expérience ensevelie). Le Maintenant du réveil, en revanche, n'est pas disponible pour le sujet historique; il suppose un kairos, l'ouverture imprévisible des portes par lesquelles le Messie peut entrer, autrement dit «l'apparition d'un lointain quelque proche que puisse être ce qui l'évoque ». Cette opposition traverse tout le Livre des passages, ainsi que les thèses sur la philosophie de l'histoire, sous la forme d'images dialectiques qui explicitent le Maintenant de la connaissabilité, tantôt de manière active, tantôt de manière passive. D'un côté, le passé est « arraché » (XVII), de l'autre il «échoit » (III) ; parfois il est question du « saut du tigre dans le passé » plein de force (XIV), à d'autres moments de la "remémoration » non violente ; de même, à la citation critique et destructive (N 11,3) fait face la citabilité rédemptrice (III); au fragment arraché, la structure monadologique de l'objet historique $(\mathrm{N} \mathrm{10,3)}$ et, de nouveau, à l'objet dans sa singularité est opposée l'apocatastase de tout le passé (N 1a, 3). Enfin, la force qui nous est donnée, bien que faible et messianique (II), est mise en regard de l'arrivée de quelque chose de plus grand qui ne peut accomplir que ce en raison de quoi celui qui écrit l'histoire (et manifestement aussi celui qui la fait?) ne peut raviver l'étincelle de l'espérance que dans le passé, non dans le futur (VI).

26 La solution à cette contradiction (qui ne m'appartient pas) n'apparaît ni dans le Livre des passages inachevé, ni dans les autres ébauches menées à leur terme. Cela pourrait, à 
mon avis, expliquer pourquoi le texte - et avec lui l'ambitieux projet poursuivi par Walter Benjamin tout au long de sa vie - n'a pas atteint la forme d'un ouvrage, et peutêtre aussi pourquoi il ne pouvait pas l'atteindre. Cela ne diminue en rien la portée de ce commentaire singulier, né dans une époque sombre, que l'on peut désormais lire comme une préhistoire de l'actuelle Fin de siècle ( « Il faut fonder le concept de progrès sur l'idée de la catastrophe. Que "les choses continuent comme avant": voilà la catastrophe ${ }^{117}$.» - N 9a, 1). De la même manière, le fait qu'un tel commentaire ait pu être écrit alors qu'une menace quotidienne pesait sur les conditions matérielles d'existence de son auteur ne peut que forcer l'admiration et plonger dans la honte celui qui à la chance d'enseigner en tant que fonctionnaire. À cet égard, ce texte qui, en dépit de la nouvelle organisation des documents de travail récemment mis en oeuvre, ne pourra pas prendre la forme d'un véritable ouvrage, laisse bel et bien derrière lui une intention résolue : "avoir sans cesse à l'esprit que le commentaire d'une réalité (c'est ce que nous écrivons ici) réclame une méthode tout à fait différente de celle appelée par le commentaire d'un texte. Dans un cas, c'est la théologie qui est la science fondamentale, dans l'autre c'est la philologie ${ }^{118}$. » (Premières notes, 0,9 ). Des éléments qu'il faudrait rappeler à ceux qui adoptent une méthode aujourd'hui dominante, pratiquant la théologie sans le soupçonner en interprétant un peu rapidement des réalités historiques comme des textes! Mais le texte de Benjamin est aussi extraordinaire en ce qu'il laisse en suspens des questions qui donnent une chance à ses successeurs de poursuivre ce qui a été ébauché, et de suivre une trace qui n'est sans doute pas à la hauteur de l'aura qu'ils ont obtenue en suivant Benjamin. Dans ce commentaire, qui entendait mettre en valeur quelques aspects, et non l'ensemble du Livre des passages, mon ambition n'était pas de concurrencer la philologie monumentale de Benjamin. C'est pourquoi je ne me suis pas préoccupé des problèmes complexes de l'histoire des textes. J'accepte volontiers les critiques et je ne justifie mes remarques que par l'inspiration profane que je dois à Benjamin - la trace qui nous rapproche d'un prédécesseur est préférable à l'apparition d'un lointain, si parfait puisse-t-il être, dans le Maintenant de la connaissabilité.

Engels, F. (1975) : La Situation de la classe laborieuse en Angleterre, d'après les observations de l'auteur et des sources authentiques [1845], trad. de G. Badia et J. Frédéric, Paris.

\section{BIBLIOGRAPHIE}

Adorno T. W (1999 [1970]) : Sur Walter Benjamin, éd. par Rolf Tiedemann, trad. par Christophe David, Paris.

Baudelaire, Ch. (1980) : Euvres complètes, éd. par Michel Jamet, Paris.

Benjamin, W. (1979) : Charles Baudelaire, Un poète lyrique à l'apogée du capitalisme, trad. par Jean Lacoste, Paris.

Benjamin, W. (1982) : Gesammelte Schriften, vol. V.2, éd. par Rolf Tiedemann et Hermann Schweppenhäuser, Francfort-sur-le-Main. 
Benjamin, W. (2000) : Euvres, t. III, Paris.

Benjamin, W. (2003 [1939]) : L'CEuvre d'art à l'époque de sa reproductibilité technique, trad. par Maurice de Gandillac, revue par Rainer Rochlitz, Paris.

Benjamin, W. (2006) : Paris, capitale du XIX siècle. Le livre des passages, trad. par Jean Lacoste, $3^{\mathrm{e}}$ édition, Paris.

Blumenberg, H. (1974) : Säkularisierung und Selbstbehauptung, Francfort-sur-le-Main.

Caillois, R. (1937) : « Paris, mythe moderne », Nouvelle revue française, 48, 1937, p. 682-699.

Caillois, R. (1972 [1938]) : Le Mythe de l'homme, Paris.

Doncourt,A. S. (1889) : Les Expositions universelles, Lille-Paris.

Gumbrecht, H.-U. et al. (éd) : Honoré de Balzac, Munich, 1980.

Habermas, J. (1987) : Théorie de l'agir communicationnel, Paris.

Hegel, G. W. F. (1979) : Leçons sur la philosophie de l'histoire, trad. par J. Gibelin, Paris : Vrin.

Hegel, G. W. F. (1995) : Cours d'esthétique, trad. par J.-P. Lefebvre et V. von Schenk, Paris : Aubier.

Hess, G. (1953) : Die Landschaft in Baudelaires « Fleurs du Mal », Heidelberg : C. Winter.

Jauss, H. R. (1977) : Ästhetische Erfahrung und literarische Hermeneutik, Munich.

Jauss, H. R. (1982) : « Norwid as Contemporaries », dans Structure of Literary Process, éd. par P.

Steiner et al., Amsterdam, p. 285-295.

Kaiser, G. (1973) : « Benjamins “ Geschichtsphilosophische Thesen” ", dans Antithesen -

Zwischenbilanz eines Germanisten, Francfort-sur-le-Main.

Maag, G. (1986) : Kunst und Industrie im Zeitalter der ersten Weltaustellungen, Munich.

Ricœur, P. (1986) : «Le modèle du texte : l'action sensée considérée comme un texte »,dans : id. : Du texte à l'action, Essais d'herméneutique II, Paris, p. 183-211.

\section{NOTES}

1. Benjamin (2006), p. 464. Les indications entre parenthèses renvoient au système de notation utilisé par Walter Benjamin et repris dans chacune des éditions du Livre des passages.

2. Ibid., p. 435.

3. Ibid., p. 447.

4. NdT : Dans la traduction française, cette indication de l'année 1798 n'apparaît plus. Voir Benjamin (1979), p. 62 : Walter Benjamin se réfère à un rapport de police de cette année (cité par Adolphe Schmidt dans Tableaux de la Révolution française) dans lequel il est écrit : « Il sera toujours presque impossible de rappeler et de maintenir les bonnes mœurs dans une population amoncelée où chaque individu, pour ainsi dire, inconnu de tous les autres se cache dans la foule, et n'a à rougir aux yeux de personne. »

5. Ibid., p. 67.

6. Ibid., p. 65 sq.

7. Caillois (1937), repris dans Caillois (1972), p. 158, cité dans Benjamin (2006), (C 8, 4).

8. Le projet de Caillois, indiqué dans sa remarque finale (Caillois [1937], p. 699, ou Caillois [1972], note 1, p. 175), n'était pas seulement d'« étudier la littérature indépendamment de tout point de vue esthétique et (...) considérer plutôt son rôle d'emprise, son conditionnement social, sa fonction de mythe en rapport avec des phases nouvelles de l'histoire des idées et de l'évolution 
du milieu ", il se référait aussi à un catalogue de questions, semblable aux rubriques présentées dans les notes de Benjamin, comme ce dernier le remarque lui-même (N 7, 1).

9. Caillois (1937), p. 698 (en lien avec « Le peintre de la vie moderne »).

10. Benjamin (2006), p. 463.

11. Benjamin (1979), p. 62, p. 84.

12. Ibid., p. 55 sq.

13. $\mathrm{NdT}$ : Cet essai s'intitule Le Peintre de la vie moderne et le chapitre en question a pour titre précis « L'Artiste, homme du monde, homme des foules et enfant ».

14. Engels (1975), p. 59-60, cité dans Benjamin (1979), p. 86.

15. Baudelaire (1980), "L'Artiste, homme du monde, homme des foules et enfant ", dans Le Peintre de la vie moderne, p. 795-796.

16. Benjamin (1979), p. 87.

17. Ibid., p. 97.

18. Ibid., p. 98.

19. Ibid., p. 175.

20. Baudelaire (1980), "L'Artiste, homme du monde, homme des foules et enfant ", dans Le Peintre de la vie moderne, p. 795. NdT : La référence bibliographique donnée par Jaussest juste, mais il remplace dans sa transcription en français les termes «incognito » par «innocence» et « amoureux » par « amateur ».

21. Benjamin (2006), p. 445.

22. Paris ou le livre des cent-et-un, Bruxelles, 1932, p. 90-103.

23. Benjamin (1979), p. 58, 59, et 60.

24. Dans Gumbrecht (1980), p. 178.

25. Voir à ce propos Jauss (1982).

26. Benjamin (2006), p. 42.

27. Baudelaire (1980), « Les Foules », dans Le Spleen de Paris, p. 170.

28. Ibid., p. 466 : « Fondamentalement, l'identification à la marchandise est une identification à la valeur d'échange. Le flâneur est le virtuose de cette identification. Il emmène en promenade le concept même de vénalité. »

29. Benjamin (1979), p. 164.

30. Ibid., p. 178.

31. Baudelaire (1980), «Les Foules », dans Le Spleen de Paris, p. 170.

32. Benjamin (2003), note 1, p. 22.

33. Ibid.

34. Blumenberg (1974).

35. Ibid., p. 13.

36. Voir Ricœur (1986).

37. Benjamin (2003), note, p. 23.

38. Habermas (1987), p. 55.

39. L'histoire intellectuelle montre que la question de la sincérité n'est devenue un enjeu social qu'à partir du temps de Montaigne et Shakespeare, et que l'authentique ne fut pas érigé en norme esthétique avant le XVIII ${ }^{\mathrm{e}}$ siècle, tout d'abord avec la prétention de Rousseau d'affirmer la vérité de son autoprésentation, même au travers de ses erreurs et de ses illusions. Cette norme s'acheva au plus tard avec la formule de Dilthey «l'expérience et la poésie » (sur ce point, voir Jauss [1977]).

40. Benjamin (2003), p. 14.

41. Ibid., p. 13.

42. Hegel (1979), p. 313, cité dans L'Euvre d'art à l'époque de sa reproductibilité technique (dernière version), dans Benjamin (2000), note 1, p. 282.

43. Ibid. 
44. Hegel (1995), t. I, p. 17, cité dans Benjamin (2000), p. 283.

45. Benjamin (2003), p. 16.

46. Benjamin (2000), p. 287.

47. Benjamin (1979), p. 200.

48. Ibid., p. 201.

49. Ibid., p. 200. Sur ce point, voir également Jauss (1977), p. 154 sq.

50. Benjamin (1979), p. 200.

51. Benjamin (2003), p. 44.

52. Ibid., p. 56.

53. NdT : Le concept d' «illumination profane» est développé par Walter Benjamin dans son article sur le surréalisme, «Le surréalisme. Dernier instantané de l'intelligentsia européenne », publié en février 1929.

54. Benjamin (1979), p. 189.

55. Ibid., note p. 190.

56. Ndt : Il s'agit d'une citation de Michelet, présentée dans Benjamin (2006), p. 36.

57. Ibid., p. 46.

58. Ibid.

59. Adorno, T. W., « Anmerkungen des Herausgebers. Zeugnisse zur Entstehungsgeschichte», dans Benjamin (1982), p. 1135.

60. Benjamin (2006), p. 41.

61. Ibid.

62. Ibid.

63. Adorno, T. W., «Anmerkungen des Herausgebers. Zeugnisse zur Entstehungsgeschichte», dans Benjamin (1982), p. 1130.

64. Benjamin (2006), p. 222.

65. Ibid.

66. Benjamin (2006), p. 223-224.

67. Ibid., p. 224.

68. Ibid., p. 36.

69. Ibid., p. 174.

70. Ibid., p. 35.

71. Ibid., p. 172.

72. Ibid., p. 36.

73. Ibid., p. 181.

74. Doncourt (1889), p. 53, cité Benjamin (2006), p. 207.

75. Benjamin (2006), p. 50, p. 219.

76. Maag (1986), p. 78 sq.

77. Baudelaire (1980), « Exposition universelle, 1855 », p. 723.

78. Ibid., p. 723-724.

79. Ibid., p. 725.

80. Ibid.

81. Ibid., p. 722.

82. Benjamin (2006), p. 907.

83. Hippolyte Babou cité dans ibid., p. 219.

84. Ibid., p. 43.

85. Cette explication complète la quatrième de mes « thèses sur la position de Baudelaire dans la modernité esthétique ", $3^{\mathrm{e}}$ colloque international W.-Krauss, dans Weimarer Beiträge, 31, 1985, p. 16-23.

86. Benjamin (2006), p. 560.

87. Barbey d'Aurevilly (J 3a, 1), Thierry (J 26, 1). 
88. Benjamin (2006), p. 43.

89. Baudelaire lui-même défendait son livre avec l'argument selon lequel on se méprendrait sur sa «terrible moralité » si on ne prenait pas en considération le principe de sa composition : «À un blasphème, j'opposerais des élancements vers le ciel ; à une obscénité, des fleurs platoniques (...). Livre destiné à représenter l'agitation de l'esprit dans le mal ». (Baudelaire [1980], «Le procès des Fleurs du mal, Notes pour mon avocat », p. 140)

90. D'après Hess (1953).

91. Benjamin (2006), p. 43.

92. Baudelaire (1980), « Peintres et aquafortistes », p. 822-823.

93. Adorno (1999), p. 116.

94. Benjamin (2006), p. 480 (souligné par H.R. Jauß).

95. Ibid., p. 563.

96. Ibid., p. 480.

97. Ibid., p. 478.

98. Voir sur ce point la quatrième de mes « Thèses sur la position de Baudelaire... » L'affirmation de Benjamin - «L'Antiquité de Baudelaire est l'Antiquité romaine. L'Antiquité grecque n'apparaît dans son univers qu'en un endroit.» (Benjamin [1979], p. 131 sq.) - réfute déjà un examen de cette thématique dans Les Fleurs du Mal. Le poème Le Cygne (prototype pour Benjamin de l'interpénétration du moderne et de l'antique) cite Andromaque ; Lesbos et Femmes damnées citent Sappho, Delphine et Hippolyte : ce qui, au-delà, peut encore apparaitre comme antique renvoie à un monde ancien mythique - dans J'aime le souvenir de ces époques nues, La vie antérieure, La Géante. La liste est déjà terminée. Le dandy comme "dernier éclat d'héroïsme dans les décadences » [ «Le Dandy ", dans Le peintre de la vie moderne, dans Baudelaire (1980), p. 807] peut difficilement le contrebalancer. Ce bilan négatif n'était pas non plus une surprise, si l'on prend la phrase provocatrice publiée dans Salut public plus sérieusement que ne le fit Benjamin, bien qu'il s'y référât à la fin de Le Paris du Second Empire chez Baudelaire: «Plus de tragédies, plus d'histoire romaine. Ne sommes-nous pas aujourd'hui plus grands que Brutus, etc. ? » [Le Salut public, n 1, 27 février 1848, dans Baudelaire (1980), p. 906]

99. Benjamin (2006), p. 116.

100. Ibid.

101. Benjamin (2006), p. 122.

102. Ibid., p. 405.

103. Ibid., p. 478.

104. Ibid., p. 479.

105. Kaiser (1973), p. 241-242 (d'après P. Szondi, « Hoffnung im Vergangenen », ibid., note 78).

106. Ibid., p. 217-218.

107. Benjamin (2000), Sur le concept d'histoire, p. 430.

108. Benjamin (2006), p. 488.

109. Benjamin (2000), Eduard Fuchs, collectionneur et historien, p. 174.

110. Ibid., Sur le concept d'histoire, p. 441.

111. Ibid., p. 428-429.

112. Ibid., p. 441.

113. Benjamin (2006), p. 475.

114. Benjamin (2000), Sur le concept d'histoire, p. 429.

115. Ibid., p. 442.

116. Kaiser (1973), p. 235.

117. Benjamin (2006), p. 491.

118. Ibid., p. 477. 
INDEX

Mots-clés : histoire de la réception, Charles Baudelaire, littérature, marxisme, Paris, XIXe siècle Schlüsselwörter : Rezeptionsgeschichte, Charles Baudelaire, Literatur, Marxismus, Paris, 19. Jahrhundert

\section{AUTEURS}

\section{HANS ROBERT JAUSS}

Hans Robert Jauss (1921-1997), théoricien de la littérature. Pour plus d'informations, voir la notice suivante. 